\title{
Avian influenza A (H5N1) infection with respiratory failure and meningoencephalitis in a Canadian traveller
}

\author{
Naheed Rajabali MD¹, Thomas Lim MD², Colleen Sokolowski MD², Jason D Prevost MD², Edward Z Lee MD²
}

\begin{abstract}
N Rajabali, T Lim, C Sokolowski, JD Prevost, EZ Lee. Avian influenza A (H5N1) infection with respiratory failure and meningoencephalitis in a Canadian traveller. Can J Infect Dis Med Microbiol 2015;26(4):221-223.

In an urban centre in Alberta, an otherwise healthy 28-year-old woman presented to hospital with pleuritic chest and abdominal pain after returning from Beijing, China. After several days, this was followed by headache, confusion and, ultimately, respiratory failure, coma and death. Microbiology yielded influenza A subtype H5N1 from various body sites and neuroimaging was consistent with meningoencephalitis. While H5N1 infections in humans have been reported in Asia since 1997, this is the first documented case of H5N1 influenza in the Western Hemisphere. The present case demonstrated the typical manifestation of H5N1 influenza but, for the first time, also confirmed previous suggestions from human and animal studies that $\mathrm{H} 5 \mathrm{~N} 1$ is neurotropic and can manifest with neurological symptoms and meningoencephalitis.
\end{abstract}

Key Words: Encephalitis; H5N1; Influenza A; Meningoencephalitis

\author{
L'infection par la grippe aviaire A ( $\mathrm{H} 5 \mathrm{~N} 1)$ \\ accompagnée d'une insuffisance respiratoire et \\ d'une méningoencéphalite chez une voyageuse \\ canadienne
}

In 1997, a poultry-originating influenza, H5N1 influenza virus, 1 caused an outbreak in Hong Kong, China. This was the first time it had been isolated from a human patient. Since then, $>600$ people have been infected with the H5N1 virus worldwide in the past 10 years, with a mortality rate of approximately $61 \%$ (1). Human $\mathrm{H} 5 \mathrm{~N} 1$ virus infection generally manifests as severe pneumonia progressing to acute respiratory distress syndrome, but can also present with abdominal pain and diarrhea $(1,2)$. It also demonstrates neurotropism, being isolated in the neurons of animals and asymptomatic individuals $(3,4)$.

\section{CASE PRESENTATION}

A healthy 28-year-old woman presented to the Red Deer Regional Hospital (Red Deer, Alberta) emergency department December 28, 2013 with a two-day history of right-sided pleuritic chest pain, mild dyspnea, nausea, right upper quadrant abdominal pain, malaise and chills. Her symptoms began as isolated chest pain during her flight back to Canada after a three-week stay in Beijing, China, and she presented to the hospital the day after her return. Her history was negative for sick contacts, exposure to poultry or birds, and visits to outdoor markets or farms. Her temperature was $39.7^{\circ} \mathrm{C}$ with a heart rate of 125 beats/min and respiratory rate of 18 breaths/min. The rest of her vital signs were normal. Her physical examination was remarkable only for mild tenderness at the right upper abdominal quadrant. Bloodwork demonstrated an elevated leukocyte count of $12.6 \times 10^{9} / \mathrm{L}$ and was negative otherwise, including blood cultures.
An electrocardiogram showed sinus tachycardia with nonspecific $\mathrm{T}$ wave inversions in leads III and aVF. Her initial chest radiograph revealed a subtle rounded consolidation of the right lung apex. A subsequent computed tomography pulmonary angiogram of the chest ruled out pulmonary embolism, but identified a dense rounded consolidation of the right lung apex with surrounding ill-defined ground-glass attenuation. Chest pain was her predominant clinical symptom, and the patient was prescribed a five-day outpatient course of levofloxacin with a diagnosis of bacterial pneumonia.

She returned to the emergency department in the afternoon of January 1, 2014 with persistent shortness of breath and worsening right-sided chest pain, without cough. She had developed a frontal headache, lightheadedness, abdominal pain and several episodes of vomiting. This time, her respiratory rate was 22 breaths/min and she required supplemental oxygen at $2 \mathrm{~L} / \mathrm{min}$ on nasal prongs to maintain an oxygen saturation of $92 \%$. She had diminished breath sounds in the right lung zones. Her abdomen was diffusely tender, but more so in the right upper quadrant and epigastric region without guarding or distention.

Investigations again revealed leukocytosis at $10.2 \times 10^{9} / \mathrm{L}$. A chest radiograph at 17:31 showed a small right pleural effusion and worsening of the right apical pneumonia. A chest tube was placed on the right, draining $300 \mathrm{~mL}$ of slightly cloudy, yellowish-tan pleural fluid. The patient's antimicrobial coverage was broadened to pipercillin-tazobactam and azithromycin for nonresolving pneumonia. The abdominal pain was attributed to the parapneumonic effusion because it resolved several hours following

${ }^{1}$ University of Alberta, Edmonton; ${ }^{2}$ Red Deer Regional Hospital, Red Deer, Alberta

Correspondence: Dr Edward Z Lee, Red Deer Regional Hospital, 431254 Avenue, Red Deer, Alberta T4N 4M1. Telephone 403-314-1435, e-mailezlee@gmail.com 


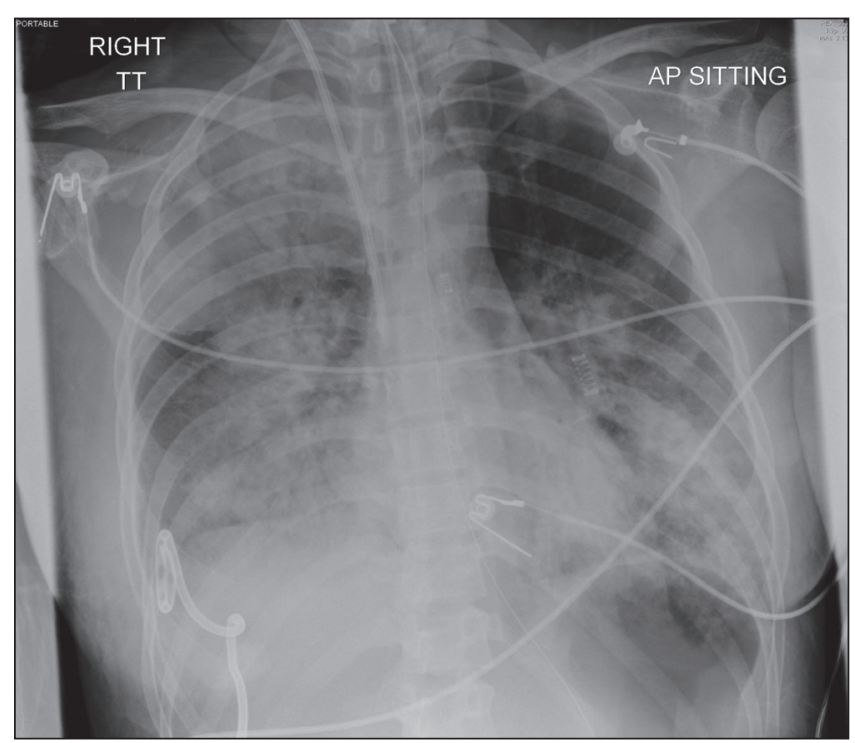

Figure 1) Chest radiograph demonstrating right greater than left dense bilateral consolidation consistent with worsening pneumonia after the patient was transferred to the intensive care unit and intubated for respiratory failure

insertion of a chest tube. The pleural fluid was exudative using Light's criteria and pathology revealed scant benign mesothelial cells with reactive changes in the background of numerous neutrophils. No bacteria were seen on Gram stain and there was no growth on cultures. An abdominal ultrasound revealed two small liver hemangiomas and was otherwise unremarkable.

On the following morning, January 2, she felt worse overall and began to notice blood-tinged sputum with her cough. By the late evening, her oxygen requirements increased to $8 \mathrm{~L} / \mathrm{min}$. The chest radiograph demonstrated progressive bilateral lung consolidation. That evening, she had a transient loss of consciousness, with recovery, followed by blurred vision that then improved. She gave a history of intermittent neck pain. A neurological examination was performed, which revealed normal extraocular eye movements, no nystagmus, pupils that were equal and reactive to light symmetrically, normal facial nerves and normal trigeminal nerve sensation. The fundi were not visualized. At this point, the intensive care unit (ICU) was consulted.

In the evening, the patient complained of increasing dyspnea and persisting sharp, right-sided chest pain, again worse with inspiration. She was alert and oriented, denied having a headache, visual changes or neck stiffness. She also denied having a sore throat or abdominal pain.

On examination by the ICU, she showed signs of respiratory distress along with tachypnea at 24 breaths/min saturating at $96 \%$ on $8 \mathrm{~L} / \mathrm{min}$ of oxygen by mask. She had normal vital signs otherwise and scored 15 on her Glasgow Coma Scale. She had an intermittent cough, productive of pink frothy sputum. She had diminished breath sounds bilaterally, but more severe throughout the right lung zones. Although alert, she was slow to answer questions and would occasionally stare away for several seconds requiring repeat prompting. She did not have other clinical features of seizure. Her responses were limited to two-word sentences. On one occasion, when she did not communicate verbally, she used a laptop computer. Although her typing motions appeared to be purposeful, the typed text was nonsensical. Otherwise, no gross neurological deficits were detected. She did not exhibit any facial droop or asymmetry, and she moved all four limbs spontaneously. She did not have meningismus.

Her diagnosis was believed to be worsening H1N1 influenza, given the recent regional H1N1 influenza outbreak. Repeat sputum cultures were sent, including samples for acid-fast bacteria and viral swabs. Oseltamivir was added at this time to the current antimicrobials.

Within $4 \mathrm{~h}$, the patient's oxygen requirements increased to $12 \mathrm{~L} / \mathrm{min}$ of oxygen by simple mask. She was alert, but markedly agitated, disoriented and confused. Again, no neck stiffness or discomfort were elicited.

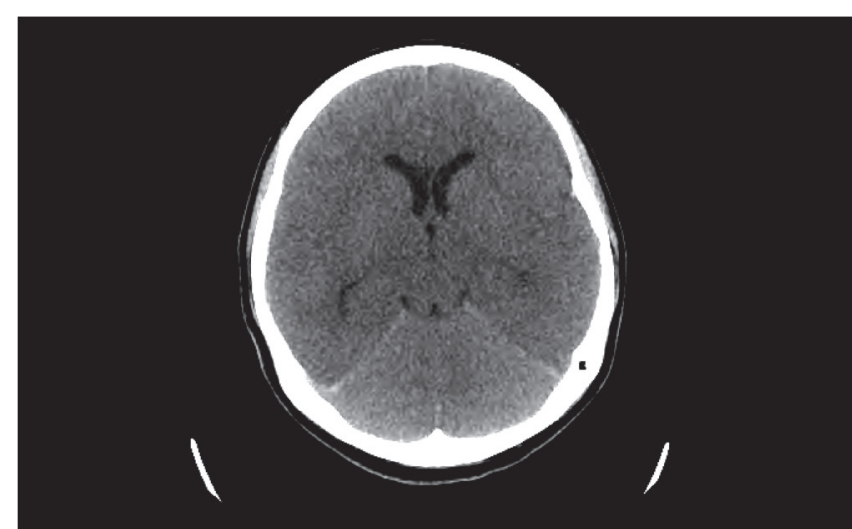

Figure 2) Computed tomography scan of the head demonstrating generalized cerebral parenchymal swelling, diffuse sulcal space and cisternal space effacement suggestive of meningoencephalitis

She was transferred to the ICU where she was sedated and intubated. Her initial ventilator settings were pressure controlled ventilation $24 \mathrm{cmH}_{2} \mathrm{O}$, positive end-expiratory pressure $12 \mathrm{cmH}_{2} \mathrm{O}$, fraction of inspired oxygen $70 \%$, peak pressure $39 \mathrm{cmH}_{2} \mathrm{O}$, respiratory frequency and respiratory rate of 22 breaths/min, with volumes of $400 \mathrm{~mL}$ to

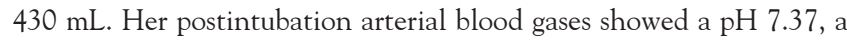
partial pressure of carbon dioxide of $36 \mathrm{mmHg}$, a partial pressure of oxygen of $83 \mathrm{mmHg}$, a bicarbonate of $21 \mathrm{mmol} / \mathrm{L}$, base deficit 4 and oxygen saturation of $93 \%$.

The chest radiograph revealed right greater than left dense bilateral consolidation, consistent with worsening pneumonia (Figure 1).

At approximately 07:00 January 3, the patient became hemodynamically unstable. She was tachycardic with heart rates varying between 120 beats/min and 150 beats/min. Her blood pressure was labile, rising to $220 / 120 \mathrm{mmHg}$. Initially, it was believed that she was inadequately sedated and her midazolam and fentanyl infusions were increased. However, her tachycardia and hypertension persisted. When examined, the patient's pupils were dilated and unresponsive to light. At this point her midazolam and fentanyl infusions were discontinued. Her blood pressure initially normalized, then over the next $1 \mathrm{~h}$ to $2 \mathrm{~h}$ she became hypotensive requiring inotropic support. She was intravenously given mannitol $20 \%$ and furosemide, and hyperventilated to a partial pressure of carbon dioxide of $30 \mathrm{mmHg}$.

A neurological examination was completed at approximately $4 \mathrm{~h}$ and $8 \mathrm{~h}$ after sedating infusions were discontinued. She was unresponsive to painful stimuli, had absent cranial nerve responses and failed the apnea test.

A nonenhanced computed tomography scan of the head (Figure 2) demonstrated generalized cerebral parenchymal swelling, diffuse sulcal space and cisternal space effacement suggestive of meningoencephalitis.

A magnetic resonance examination of the brain (Figure 3) was obtained, confirming diffuse sulcal space and cisternal space effacement with diffusely increased signal of the extra-axial cerebrospinal fluid (CSF) spaces and ependyma of the lateral ventricles on fluidattenuated inversion recovery imaging. The increased signal of the CSF spaces on fluid-attenuated inversion recovery imaging indicated proteinaceous debris within the CSF spaces and along the ependymal surface. Diffuse parenchymal swelling and slightly increased T2-weighted signal of the cortex of the temporal lobes, insular cortex and hippocampal regions was noted bilaterally, suggestive of encephalitis. Gadolinium-enhanced magnetic resonance angiography revealed markedly delayed intracranial arterial flow, with partial opacification of the anterior cerebral, middle cerebral and posterior cerebral arteries at 4 min postcontrast injection. Brisk flow to the scalp vessels was noted. Enhancement of the meninges was not observed, likely secondary to diminished intracranial blood flow.

The neurologist on call examined the patient $11 \mathrm{~h}$ after sedating medications had been discontinued. The abnormal findings of the earlier 
neurological examination were confirmed. The patient met the clinical criteria for brain death and was pronounced dead. A lumbar puncture was performed following brain death and before discontinuation of cardiorespiratory life support.

Samples from the nasopharyngeal swab, endotracheal aspirates and CSF tested positive for influenza A type H5N1. CSF showed 2+ white blood cells, $2+$ red blood cells, without bacteria seen. Details of diagnostic specimen analysis and virological investigations were reported by Fonseca (5). Autoimmune serologies obtained later returned positive for perinuclear antineutrophil cytoplasmic antibody. Myeloperoxidase and antiglomerular basement membrane antibodies were both negative.

\section{DISCUSSION}

We report a fatal case of influenza A (H5N1) in a woman, 28 years of age, who presented in January 2014 with pleuritic chest pain, dyspnea and right upper quadrant abdominal pain due to pneumonia and a pleural effusion, followed by respiratory failure, agitation, confusion and coma, after a three-week visit to Beijing, China. She was diagnosed with acute respiratory distress syndrome, secondary to pneumonia and brain death due to meningoencephalitis. The diagnosis of $\mathrm{H} 5 \mathrm{~N} 1$ was established by isolating the virus from nasopharyngeal swabs, broncho-alveolar lavage and CSF.

Our patient's demographic is in keeping with what is commonly found in humans infected with H5N1. Most patients with influenza A (H5N1) virus infection have been previously healthy. The median age of patients with influenza A (H5N1) virus infection is approximately 18 years, with $90 \%$ of patients $\leq 40$ years of age. Moreover, increases in human cases of influenza A (H5N1) have been observed during cooler months in association with increases in outbreaks among poultry $(1,6-8)$.

Unique to our experience is that this is the first reported case of $\mathrm{H} 5 \mathrm{~N} 1$ influenza in the Western world because most cases remain endemic in Asia, the Middle East, Europe and Africa. Furthermore, until now, no cases of influenza A (H5N1) illness have been identified among short-term travellers visiting countries affected by outbreaks among poultry or wild birds (9).

Our patient's initial respiratory manifestation is a common finding in H5N1-infected humans, with the virus primarily affecting the lower respiratory tract causing respiratory failure. Diarrhea is also a common presenting feature of $\mathrm{H} 5 \mathrm{~N} 1$, occurring in up to $70 \%$ of patients. Besides these common presentations, patients have also been noted to complain of vomiting and abdominal pain. These symptoms correlate with findings of viral RNA in fecal samples and viral replication in intestines $(1,2,4,10)$. Our patient complained of vomiting and crescendodecrescendo abdominal pain, but did not have diarrhea.

Our patient also had mild intermittent headaches, confusion and demonstrated symptoms and a clinical course in keeping with

\section{REFERENCES}

1. Abdel-Ghafar AN, Chotpitayasunondh T, Gao Z, et al. Update on avian influenza A (H5N1) virus infection in humans. N Engl J Med 2008;358:261-73.

2. Gambotto A, Barratt-Boyes S, de Jong M, et al. Human infection with highly pathogenic H5N1 influenza virus. Lancet 2007;371:1464-75.

3. Shinya K, Makino A, Hatta M, et al. Subclinical brain injury caused by H5N1 influenza virus infection. J Virol 2011;10:5202-7.

4. Gu J, Xie Z, Gao Z, et al. H5N1 infection of the respiratory tract and beyond: A molecular pathology study. Lancet 29;370:1137-45.

5. Fonseca K. Fatal avian influenza A(H5N1) infection in a Canadian traveler. Promed. <www.promedmail.org, archive no. 20140112.2167282> (Accessed on January 12, 2014.)

6. Park AW, Glass K. Dynamic patterns of avian and human influenza in east and southeast Asia. Lancet Infect Dis 2007;7:543-8.

7. Ortiz JR, Wallis TR, Katz MA, et al. No evidence of avian influenza A (H5N1) among returning US travelers. Emerg Infect Dis 2007;13:294-7.

8. Kaplan B, Webby R. The avian and mammalian host range of highly pathogenic avian H5N1 influenza. Virus Res 2013;178:3-11.

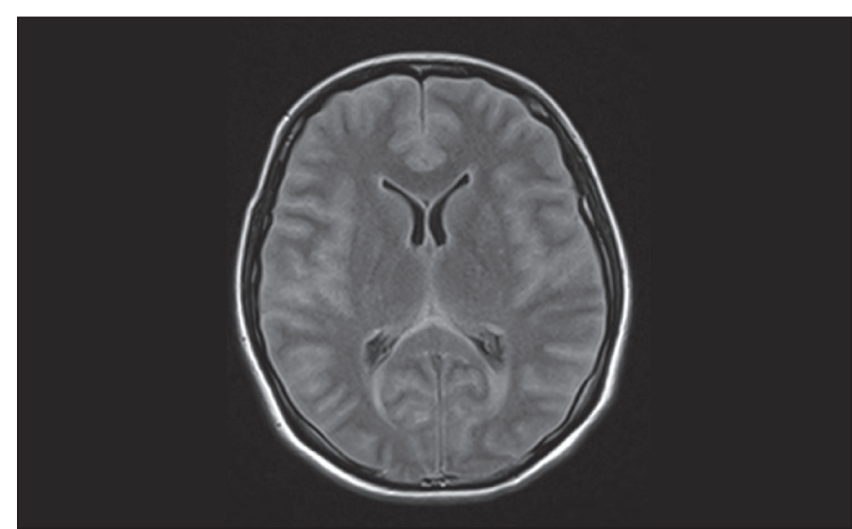

Figure 3) Magnetic resonance examination of the brain revealing diffuse sulcal space and cisternal space effacement with diffusely increased signal of the extra-axial cerebrospinal fluid spaces and ependyma of the lateral ventricles on fluid-attenuated inversion recovery imaging. Diffuse parenchymal swelling and slightly increased T2-weighted signal of the cortex of the temporal lobes, insular cortex and hippocampal regions noted bilaterally suggests encephalitis

meningoencephalitis. Encephalopathy and encephalitis in humans have been reported in cases associated with seasonal influenza $A$ and $B$ viruses $(11,12,13)$. Only one case of human central nervous system involvement has been suggested by a patient who developed a coma from whom H5N1 was isolated from CSF (10) despite being noted in mammals such as ferrets, mice and felids (8). With respect to the child who developed a coma, imaging of the brain or histological analysis was not performed, and it is uncertain whether the patient experienced encephalopathy or true encephalitis. These reports suggest the H5N1 virus is becoming more neurologically virulent and adapting to mammals. Despite the trend in virulence, the mode of influenza virus transmission remains elusive to date. It is unclear how our patient acquired the H5N1 influenza infection because she did not have any known contact with any animals or poultry.

The nonspecific clinical presentation of $\mathrm{H} 5 \mathrm{~N} 1$ influenza virus has frequently led to underdiagnoses of subsequently confirmed cases (1). Infection with H5N1 influenza virus should be considered in the differential diagnosis for patients with epidemiological risk factors presenting with systemic manifestations, including the common respiratory and gastrointestinal symptoms, clusters of intermittent abdominal pain and headache, as well as signs of meningoencephalitis.

Having a high index of suspicion for H5N1 infection is essential to the appropriate management of the illness, because treatment with antiviral agents is likely to be beneficial only when it is started early in the course of illness $(1,2,14)$.

9. World Health Organization. Antigenic and genetic characteristics of zoonotic influenza viruses and development of candidate vaccine viruses for pandemic preparedness. Wkly Epidemiol Rec 2014; 89:105-16. < www.who.int/wer/2014/wer8911.pdf> (Accessed on March 16, 2014).

10. de Jong M, Van Cam B, Tu Qui P, et al. Fatal avian influenza A (H5N1) in a child presenting with diarrhea followed by coma. N Engl J Med 2005;352:686.

11. Morishima T, Togashi T, Yokota S, et al. Encephalitis and encephalopathy associatedwith an influenza epidemic in Japan. Clin Infect Dis 2002;35:512-7.

12. Steininger C, Popow-Kraupp T, Laferl H, et al. Acute encephalopathy associated with influenza A virus infection. Clin Infect Dis 2003;36:567-74.

13. Maricich SM, Neul JL, Lotze TE, et al. Neurologic complications associated with influenza A in children during the 2003-2004 influenza season in Houston, Texas. Pediatrics 2004;114:e626-e33.

14. Liu Q, Liu D, Yang Z, et al. Characteristics of human infection with avian influenza viruses and development of new antiviral agents. Acta Pharmacologica Sinica 2013;34:1257-69. 


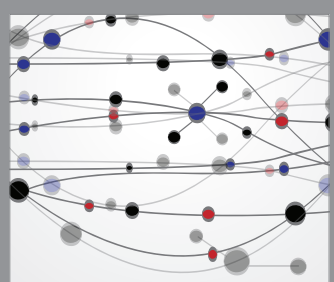

The Scientific World Journal
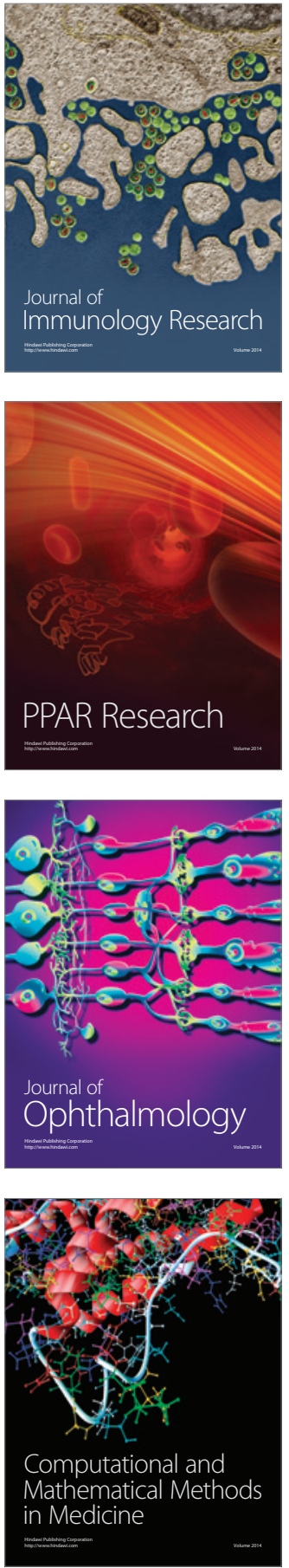

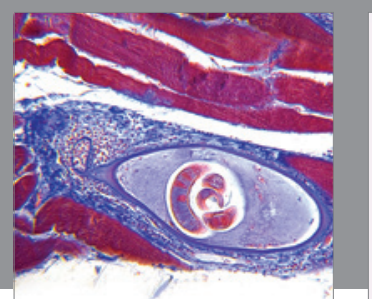

Gastroenterology Research and Practice

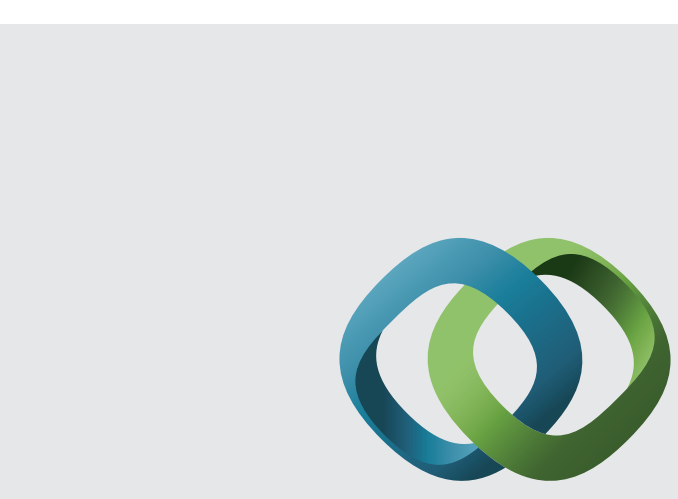

\section{Hindawi}

Submit your manuscripts at

http://www.hindawi.com
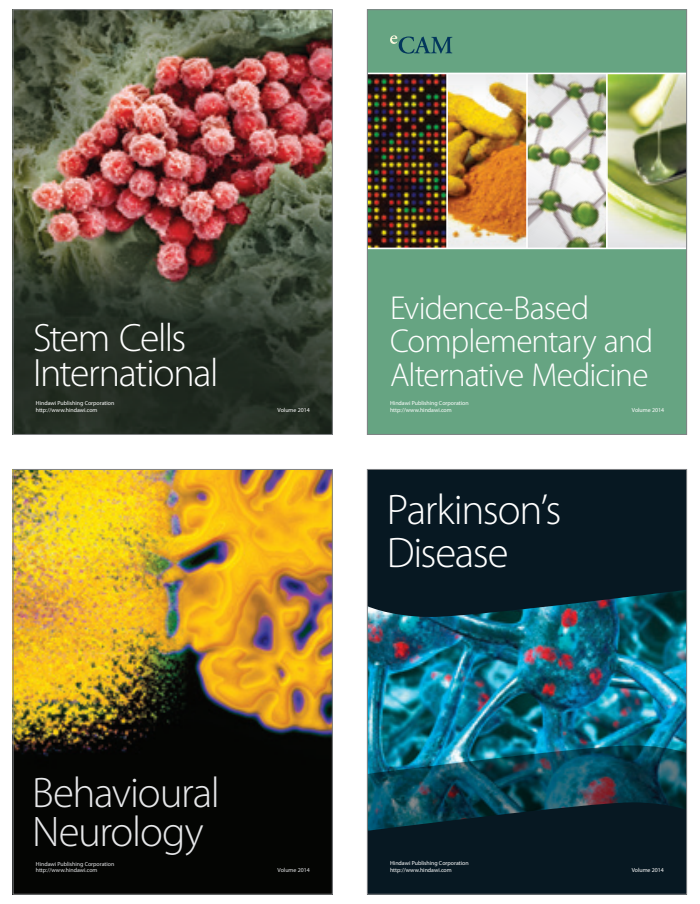
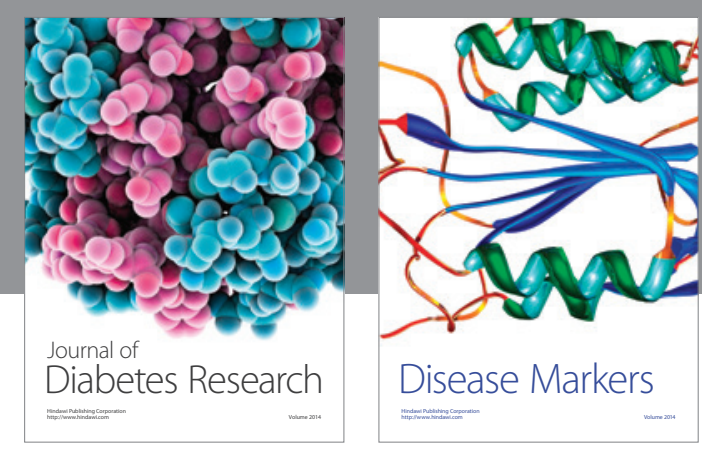

Disease Markers
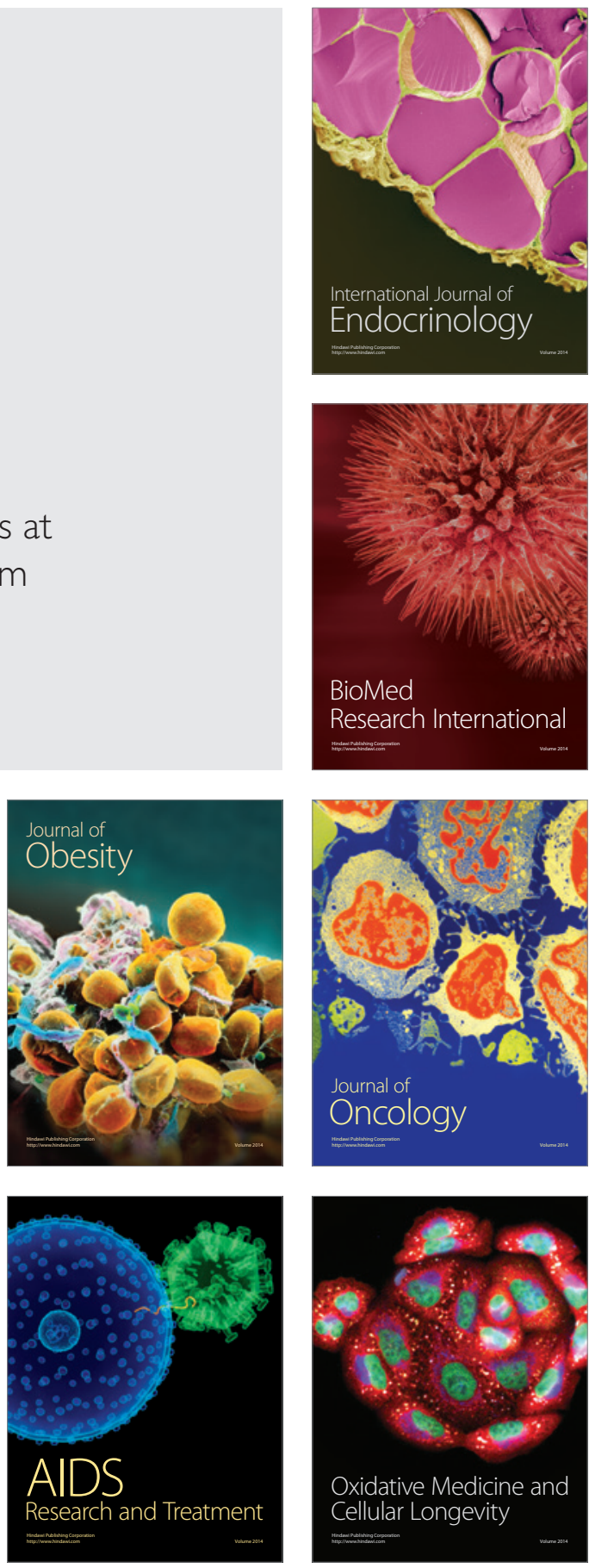